International Journal of Pure and Applied Mathematics

Volume 88 No. 4 2013, 577-592

ISSN: 1311-8080 (printed version); ISSN: 1314-3395 (on-line version)

url: http://www.ijpam.eu

doi: http://dx.doi.org/10.12732/ijpam.v88i4.12

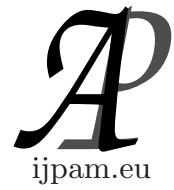

\title{
CONTROLLED TRUST FORCE INFLUENCE ON AUTOMATIC BONE DRILLING PARAMETERS IN THE ORTHOPEDIC SURGERY
}

George Boiadjiev ${ }^{1 \S}$, Kamen Delchev ${ }^{2}$, Tony Boiadjiev ${ }^{3}$, Kazimir Zagurski ${ }^{4}$, Rumen Kastelov ${ }^{5}$, Vladimir Vitkov ${ }^{2}$

${ }^{1}$ Analytical Mechanics Department

Faculty of Mathematics and Informatics

Sofia University

5, J. Boucher Blvd., 1164, Sofia, BULGARIA

${ }^{1,2}$ Institute of Mechanics

Bulgarian Academy of Sciences

Acad. G. Bonchev St., Bl. 4, 1113, Sofia, BULGARIA

${ }^{3}$ Institute of Information and Communication Technologies

Bulgarian Academy of Sciences

Acad. G. Bonchev St., Bl. 2, 1113, Sofia, BULGARIA

${ }^{4}$ Institute of System Engineering and Robotics

Bulgarian Academy of Sciences

Acad. G. Bonchev St., Bl. 2, 1113, Sofia, BULGARIA

${ }^{5}$ Orthopaedic Trauma Department

University Hospital for Emergency Medicine "Pirogov"

3-rd Orthopaedic Trauma Clinic

21 Totleben Blvd., 1606, Sofia, BULGARIA

\begin{abstract}
Background. Many orthopaedic operations involve drilling before the insertion of screws into the bone. Usually drilling is executed manually, which brings problems. Bone overheating is the most important one. To avoid such problems and reduce the subjective factor, automatic drilling is recom-

Received: October 9, 2013

(C) 2013 Academic Publications, Ltd. url: www.acadpubl.eu
\end{abstract}

$\S$ Correspondence author 
mended.

Methods. As lots of parameters influence to the drilling process, the methods are experimental. They concern the experimental identification of the drilling technical parameters including the bone resistant force and the drilling process temperature. The resistant force and temperature are measured and plotted.

Results. Specific drilling effects are revealed during the experiments. The temperature deviations are kept in the safe borders. The temperature reduction is achieved by controlled trust force during automatic bone drilling regime in comparison with hand-drilling one. Algorithms are created and their software realization is made. Curves of resistant force and temperature with respect of the time are presented. A proposed idea of ultrasonic vibration using for ultrasonically-assisted drilling of a cortical bone and applying a controlled trust force during automatic bone drilling regime is discussed.

Conclusions. Automatic bone drilling can solve the high-temperature problems which arise during manual drilling. An experimental setup is designed to identify some parameters of bone drilling such as the resistant force due to variable bone density, the appropriate mechanical torque of drilling, the linear speed of the drill, and the electromechanical characteristics of motors, drives and corresponding controllers. The automatic drilling guarantees higher safety for the patient.

AMS Subject Classification: $92 \mathrm{C} 50$

Key Words: automatic bone drilling, temperature minimization, experiments, orthopedic surgery

\section{Introduction}

Many researches have been recently devoted to robot application in orthopaedic surgery. To overcome the inaccuracy of hand-controlled positioning of orthopaedic surgical tools, a number of robotized systems have been developed during the last decade. In orthopaedic surgery it often requires the use of electrical instruments - cutting and drilling machines, saws and so on, which the surgeon holds in his hands during manipulation. By the literature reports drilling devices are used in approximately 95\% of post-trauma interventions. This illustrates the persistent interest of researchers in such manipulations. Since orthopaedic screws are often implanted in bones, one needs to perform 
bone drilling beforehand. Yet, manual drilling tends to produce such problems as apertures larger than needed, tendon or blood vessel break, overheating, etc., which affect the accuracy and safety of the operation. Bone overheating is the most important one between them.

Bone is a poor conductor of heat, with the thermal conductivity of fresh cortical bone in the region of $0.38 \pm 2.3 \mathrm{~J} / \mathrm{msK}[1,2]$. The stainless steel the drill bits usually made of, for comparison, is a much better conductor of heat than bone, having a conductivity of $14 \mathrm{~J} / \mathrm{msK}$.

However, some of the heat generated during drilling may be partially dissipated by the presence of blood and tissue but the temperature rise at the cutting edge in a deep cortical hole could be significantly high [3]. If bone is exposed for longer than $30 \mathrm{~s}$ at $50^{\circ} \mathrm{C}$ cellular necrosis will be induced [2]. When epithelial cells are exposed to a temperature of $70^{\circ} \mathrm{C}$ they will be damaged immediately, that when they are exposed to a temperature of $55^{\circ} \mathrm{C}$ for $30 \mathrm{~s}$ the result will be the same and at $45^{\circ} \mathrm{C}$ harmful effects will occur after $5 \mathrm{~h}[4,5]$.

In general, the literature shows that if the temperature rises above $55^{\circ} \mathrm{C}$ for a period of longer than one-half a minute, serious damage will be done to the bone, which may take several weeks to fully recover.

In $[6,7]$ it is shown that cutting speed had very little effect on temperature change above $55^{\circ} \mathrm{C}$ but that the force applied to the drill caused a much greater temperature increase. An idea to reduce the force applied to the drill is the usage of ultrasonic vibration for ultrasonically-assisted drilling of a cortical bone, proposed in [10]. Even the design of a micro vibration robot for orthopaedic surgery was proposed in [11], which has a sensor for torque-current connected to the end-effector and a control feedback loop.

Insofar as can be established from the literature, attempts at temperature measurement when drilling cortical bone have been carried out by placing thermocouples into the bone at given distances from the drilling area. Indifferent and sometimes contradictory results have been achieved using these methods. Because of the poor thermal conductivity of bone, its structural inconstancy and the great difficulty in modelling for heat-transfer purposes, embedding thermocouples in the bone adjacent to the drilling operation is not a satisfactory method of measuring the temperature effects [3]. An excessive temperature increase in the vicinity of the drill hole results in bone thermal necrosis which causes irreversible changes in the structure and physical properties of the bone [8]. In this case, the screw loosens reducing screw fixation stability and strength, and the necrotic tissue hampers healing and is infection-conductive [9]. All this affects patients' safety, which is of crucial importance.

Based on the literature sources, it can be concluded that if temperatures 
greater than $45^{\circ} \mathrm{C}$ are reached then the temperature at the drill-bit cutting edge must be considerably higher with consequent severe damage to the bone surface of the hole. In the same time the temperature increased as the depth of the hole increased. Speeds of 800-1400 rpm should be used and such a drilling mode provides the best cutting conditions and maintaining temperatures at a manageable level [3].

Such a drilling mode can be successfully realized by the robot application in the orthopaedic surgery, which also assures high precision and accuracy of manipulations and that all reflects to the patient's safety.

The aim of the paper is to present the experimental evidence of the advantages of automatic bone drilling concerning the temperature reduction and resistant force reduction for the sake of controlled trust force execution, confirming these advantages, discuss and analyse the temperature effects occurring during bone drilling.

\section{Experimental Setup}

We modified and improved the developed the experimental set up presented in $[12,13]$. Our experimental approach was chosen in view of the complex character of the object under study. As it was mentioned above, modelling was very difficult to accomplish since many parameters including unknown ones had to be taken into account. Yet after performing many experiments it was concluded that a robot for automatic bone drilling should incorporate components capable of guaranteeing:

- Torque up to $1.5 \mathrm{Nm}$;

- Force along the translation up to $100 \mathrm{~N}$;

- Force sensor range up to $100 \mathrm{~N}$;

- Temperature not over $52^{\circ} \mathrm{C}$;

- Accuracy less or equal to $0.5 \mathrm{~mm}$ for the drilling depth or length of the bone hole.

At the same time, robot dimensions and mass should be as small as possible.

Note that some parameters of a bone drilling operation can be experimentally identified, for example, the values of some parameters, based on the experimental evidence. Yet those very values which were obtained experimentally varied when the drill bit was replaced by a sharpened one. Hence, problems 


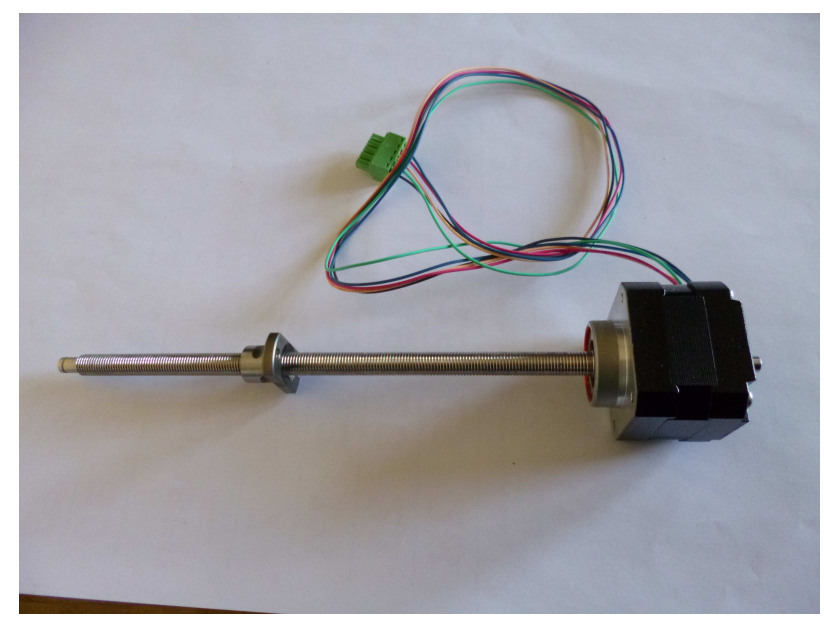

Figure 1: Linear actuator $43000-17\left(1.80^{\circ} / \mathrm{step}, 0.0158 \mathrm{~mm} / \mathrm{step}\right)$

occurred even during the course of some of the experiments, proving that the experimental setup also needs improvement.

\section{Components Mounted in the Experimental Setup}

Linear actuator 43000-17 (Figure 1). It is a stepper motor with an embedded screw for linear motion, highly accurate at low speed, small in size, translation of $1 \mathrm{~mm}$ for 4032 micro steps.

Brushless DC motor MAXON (Figure 2).

Such motors have many advantages: better speed-versus-torque characteristics, high dynamic response, high efficiency, long operating life, noiseless operation, higher speed ranges, tough structure. Note also the large torque despite the small motor size. Hence the space and weight are factors guaranteeing torques of up to $1.5-1.7 \mathrm{Nm}$.

The controllers comprise two main components: a controlling device and a power drive with built-in PID-regulators for positioning and speed. They are:

- Controller / Driver TMCM-1110 to control a linear actuator supplied with the one-axis stepper motor 43000-17.

- Servo Controller / Driver for BLDC MAXON. 


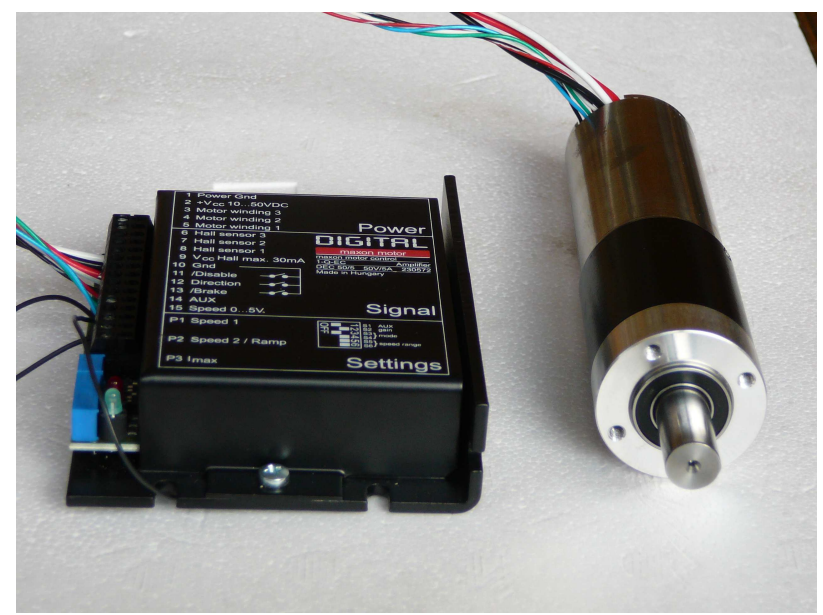

Figure 2: Brushless DC motor MAXON with servo controller/driver 1-Q-CE Amplifier DEC 50-5

The sensor system comprises:

Force sensor LMB-A-200N (Kyowa) for the bone resistant force (Figure 3).

Temperature sensor SP i-tec 2005D, an infrared non-contact temperature measuring instrument.

A Test Performance is Presented Below (Figure 5), where a beef bone is fixed.

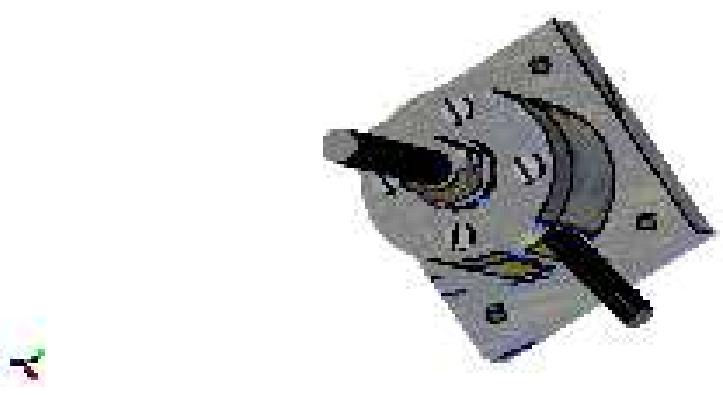

Figure 3: Force sensor LMB-A-200N (Kyowa) 


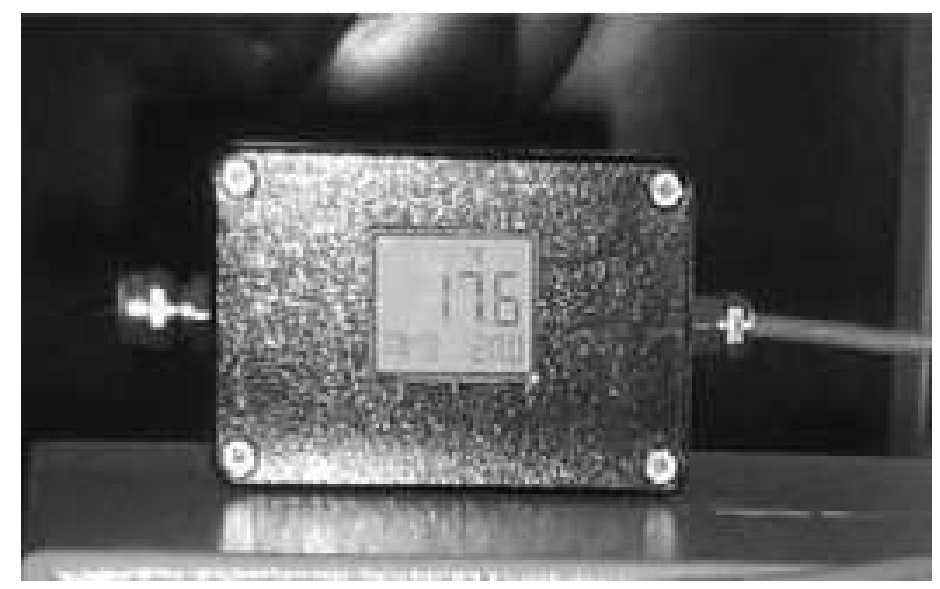

Figure 4: Temperature sensor SP i-tec 2005D
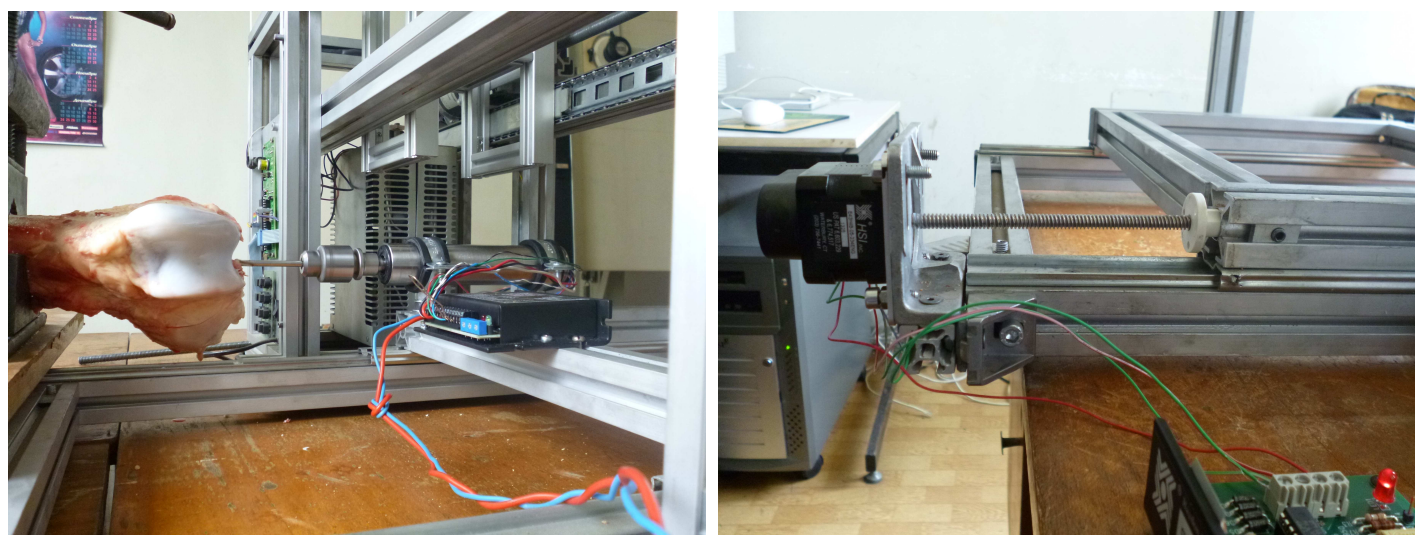

Figure 5: Drilling execution

\section{Control Algorithms}

Control algorithms are executed in the specific program environment TMCLIDE. The commands can be executed immediately after their input or the program can be downloaded in the controller to perform autonomous execution of commands.

In each cycle, the program recognizes the current state, executes the corresponding algorithm and decides the transition to the next state according to preliminarily specified criteria.

The algorithm which is followed for the current calculation of the "next tar- 


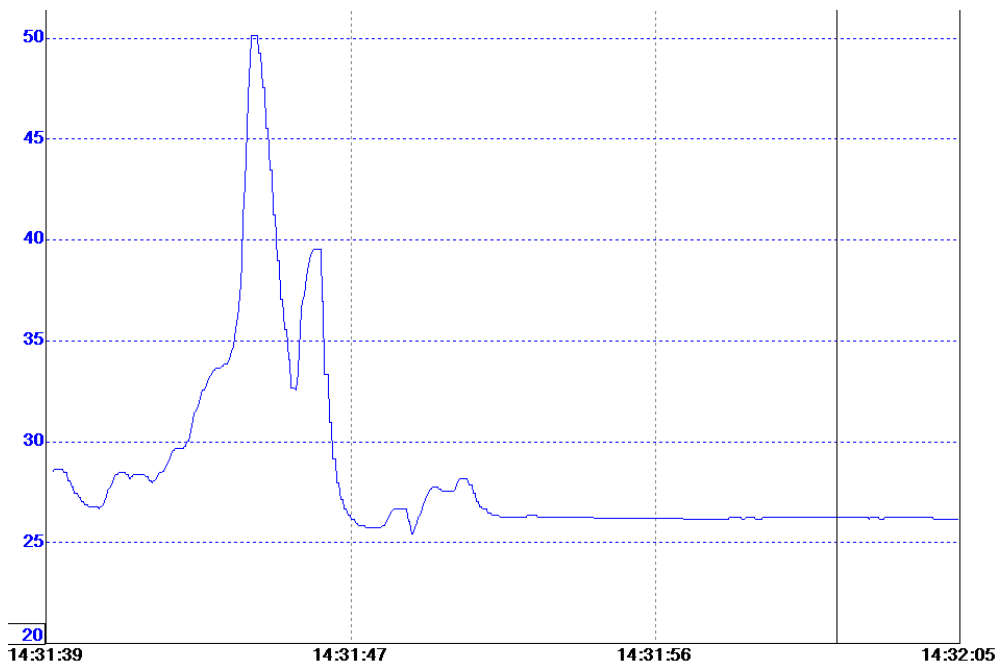

Figure 6: Temperature during manual drilling throughout the entire bone (drill bit $\varnothing 6 \mathrm{~mm}$ )

get position" of the stepper motor depends on the force sensor data. The actual force sensor data are compared to previously given values to check whether the safety requirements to prevent exceeding the limit value are being kept.

An algorithm to identify the value of the resistant force initiated during drilling is executed [13]. This value is related to the manipulation characteristics of the object and the diameter of the drill bit. The value found is used to decide whether to end bone cortex drilling.

\section{Results}

Certain effects are discussed which occur under an automatic bone-drilling regime and can be registered only during the course of an experiment. On fig. 6 and fig. 7 the temperature during manual and automatic drilling throughout the entire bone are presented. The vertical axis shows the temperature and the horizontal axis shows the time. Under the same experimental conditions the maximal value reaches $50^{\circ} \mathrm{C}$ and $42^{\circ} \mathrm{C}$ during drilling time $15 \mathrm{~s}$ and $25 \mathrm{~s}$, correspondingly. 


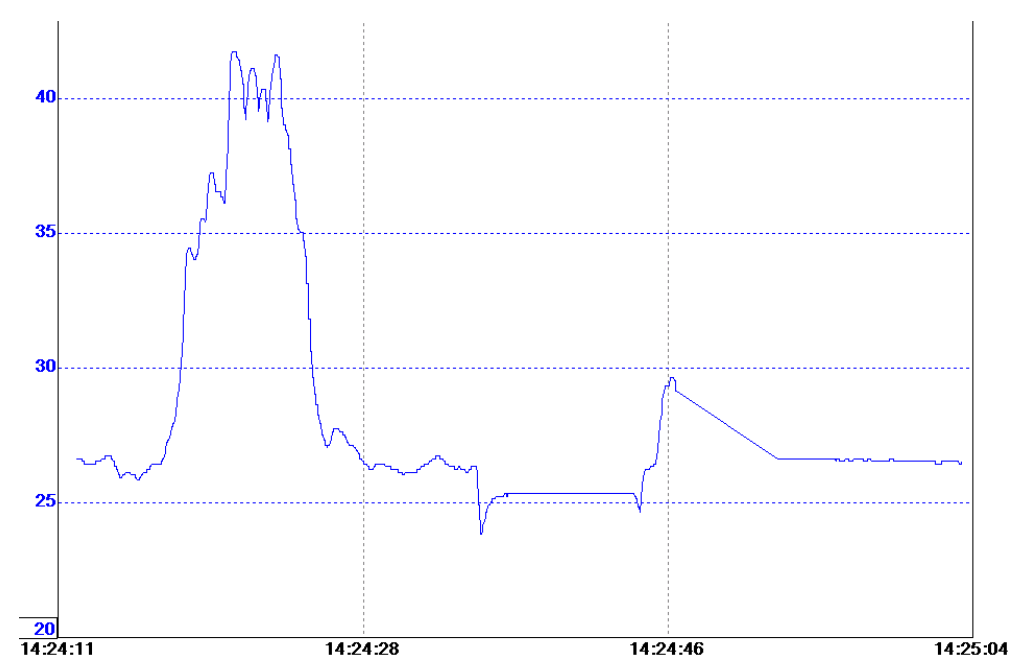

Figure 7: Temperature during automatic drilling throughout the entire bone $(2 \mathrm{~mm} / \mathrm{s}$; drill bit $\varnothing 6 \mathrm{~mm})$

\section{Accuracy and Safety}

There is a great variation in the hardness of the different bones in a particular body and there can be a great variation in the hardness of a particular bone due to the age, sex, race or medical status of the person. This variation will cause a consequent variation in the thrust, torque and power values of a particular drilling operation. Because of that, at the beginning, the resistant force is identified for a specific object (patient), taking into account the patient's characteristics [14]. The resistant force is presented on fig.8. The experimental data are smoothed. The best results are obtained when approximation polynomials of the $19^{\text {th }}$ and $21^{\text {st }}$ degree are used, respectively. We use these polynomials to evaluate the force limits not to be exceeded during drilling. Figure 8 shows that the span between the numerical values and the measurement data is relatively large, which may disturb the algorithm and program execution. The approximations help to find the type and size of appropriate filtration, recognize the local maximums and their number, i.e. recognize both cortices. The estimation of resistance force limits prevents a specific bone from possible crushing. The same approach is applied to hole depth and bone cortex size. 

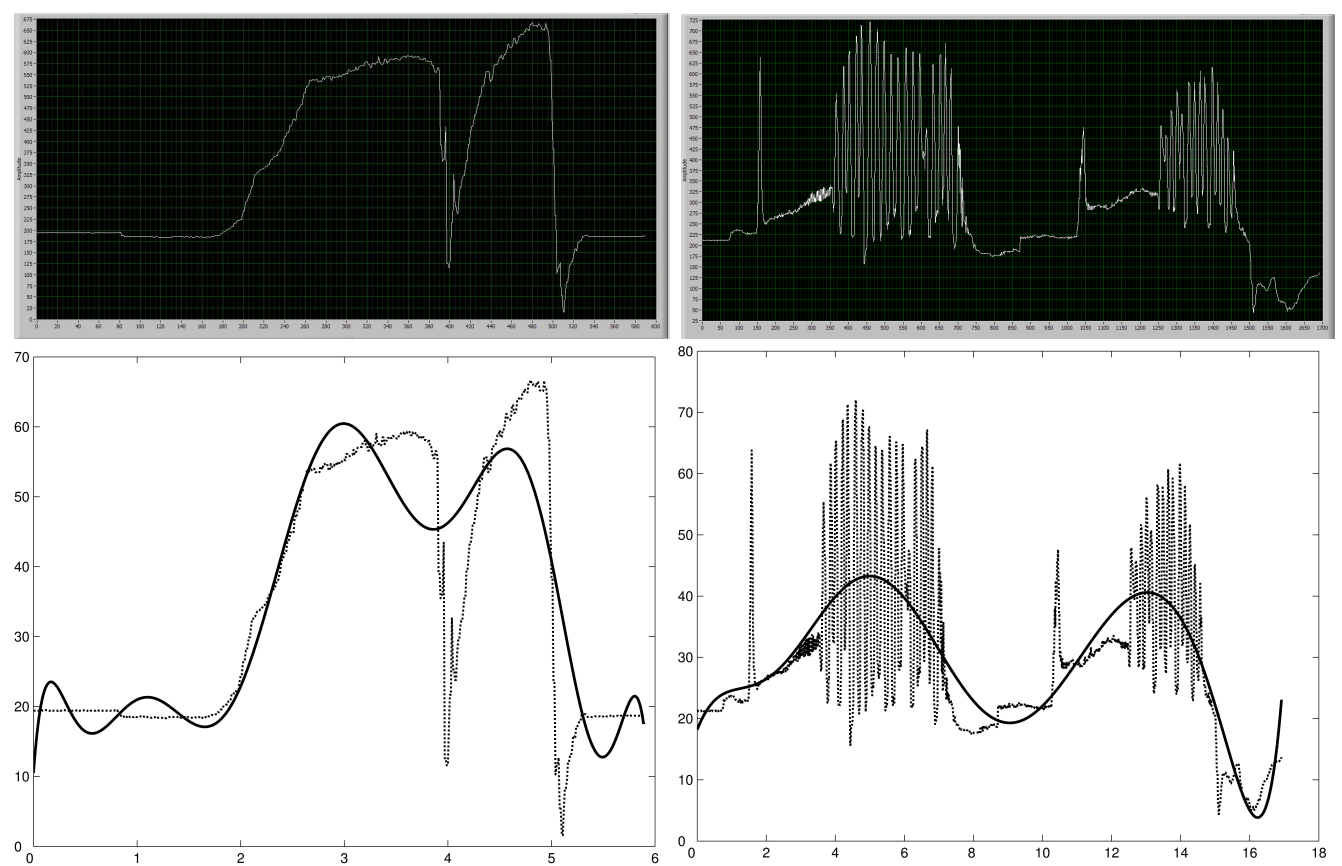

Figure 8: Approximation of the resistant force by $19^{\text {th }}$ degree polynomial function

\section{Experimental Results}

By means of the designed setup various experiments have been performed. The drilling mode included the following monitored parameters:

- Time $[\mathrm{s}]$;

- Linear velocity $[\mathrm{mm} / \mathrm{s}]$;

- Angular velocity [rpm];

- Resistant force $[\mathrm{N}]$;

- Temperature $\left[\operatorname{deg}^{\circ} \mathrm{C}\right]$.

The figures above illustrate the resistant force deviation. At the beginning its value corresponds to that of the force needed to drill the first cortex. When the cutting screw reaches the marrow, the force drops down. The resistant force is again initiated when the cutting screw starts drilling the rear cortex. 


\section{Comparison with the Theoretical State}

Nevertheless the theoretical modeling of the bone is very difficult to accomplish as it was mentioned above, some attempts were made, and for instance in [15], but here we would illustrate the numerical solution of the thermal conductivity equation and comprise it with the obtained experimental results.

It is well known, for instance in [16], the thermal conductivity equation has the form

$$
\frac{\partial u}{\partial t}=a^{2}\left(\frac{\partial^{2} u}{\partial x^{2}}+\frac{\partial^{2} u}{\partial y^{2}}+\frac{\partial^{2} u}{\partial z^{2}}\right)
$$

where for one considered body:

$a^{2}=\frac{k}{\gamma \rho}, k$ - thermal conductivity coefficient of the body, $\rho$ - its density, and $\gamma$ - the thermal capacity coefficient;

$u=u(M, t)$ - the temperature of a point $M$ of that homogeneous body, bounded by a surface $S$, in a moment of the time $t$.

In one-dimensional situation as the drill bit could be assumed to be considered, (1) is simplified to the form

$$
\frac{\partial u}{\partial t}=a^{2} \frac{\partial^{2} u}{\partial x^{2}}
$$

where the $O x$-axis is oriented along the drill bit length.

In the next it will be assumed that on the drill bit top the neat is generated which is generally described by a function $\phi(t)$ of the time $t$, which concrete type can be obtained by experimental measurements. We are looking for the temperature distribution along the drill bit. Obviously it is in relation of the drill depth, which depends on the drilling mode at last. So, the problem leads mathematically to the Caushy task for the equation (2) with initial and boundary conditions (3) and (4) respectively:

$$
\begin{gathered}
u(x, 0)=f(x), \\
u(0, t)=\phi(t) .
\end{gathered}
$$

Then the solution of the equation (2) can be written as

$$
u(x, t)=\frac{1}{2 a \sqrt{\pi t}} \int_{0}^{\infty} f(\xi)\left[e^{-\frac{(\xi-x)^{2}}{4 a^{2} t}}-e^{-\frac{(\xi+x)^{2}}{4 a^{2} t}}\right] d \xi
$$




$$
+\frac{1}{2 a \sqrt{\pi t}} \int_{0}^{\infty} \phi(\eta) e^{-\frac{x^{2}}{4 a^{2}(t-\eta)}(t-\eta)^{-\frac{3}{2}}} d \eta .
$$

Further let by "l" the drill depth is denoted. For simplicity it will be assumed the heat flow moves from the drill bit to the bone without losses and on the border between them the temperature is the same. Really, the more complex cases could be taken into account varying the coefficients of the thermal capacity and conductivity of the bodies. Under these assumptions, making the notation $u_{*}<42^{\circ} \mathrm{C}$ as an upper limit of the temperature which must not be exceeded for the sake to avoid the necrosis, we reach the following inequality:

$$
\max _{x, t} u(x, t) \leq u_{*}, \text { where } x \in[0, l], t \in[0, T]
$$

The last inequality can be considered as a criterion for the control based on the temperature effects evaluation during the automatic bone drilling. There such a criterion could be made weaker, i.e. the value $u_{*}$ could be reduced aiming to assure more safety as well as the upper borders of the functions $f(x)$ and $\phi(t)$ could be evaluated.

Bellow an example is shown illustrating the temperature distribution along the drill depth in dependence of the time. There the following values are taken: $a=1, T=40 \mathrm{~s}, l=0.04 \mathrm{~m}$. The functions $u(x, 0)$ and $u(0, T)$ have the form:

$$
\begin{aligned}
& u(x, 0)=f(x)= \begin{cases}u_{0}+\Delta u, & 0 \leq x<x_{1}, \\
u_{0}, & x_{1} \leq x<l,\end{cases} \\
& u(0, t)=\phi(t)= \begin{cases}u_{0}+\Delta u, & 0 \leq t<T, \\
0, & T \leq t<\infty .\end{cases}
\end{aligned}
$$

Here $u_{0}$ is the initial temperature at the process starting and $\Delta u$ is the temperature on the cutting edge, i.e. on the top of the drill bit. For the example they are taken as constant values $20^{\circ}$ and $50^{\circ}$ respectively. The graphics is shown on the fig. 9. On the vertical axis is the temperature and the horizontal axes show the drill depth and the time according the example.

The numerical solution of the equation (2), presented by (5), is obtained by $M A T L A B$, where $x_{1}$ has a value $3 \mathrm{~mm}$. It can be seen the temperature functional values are varying continuously depending on the coefficients as well as the initial and boundary conditions and the maximal values are placed on the border of the body.

In the example the value $70^{\circ}$ is taken like an initial condition aiming to be as much as possible closer to the result obtained by measurements using 


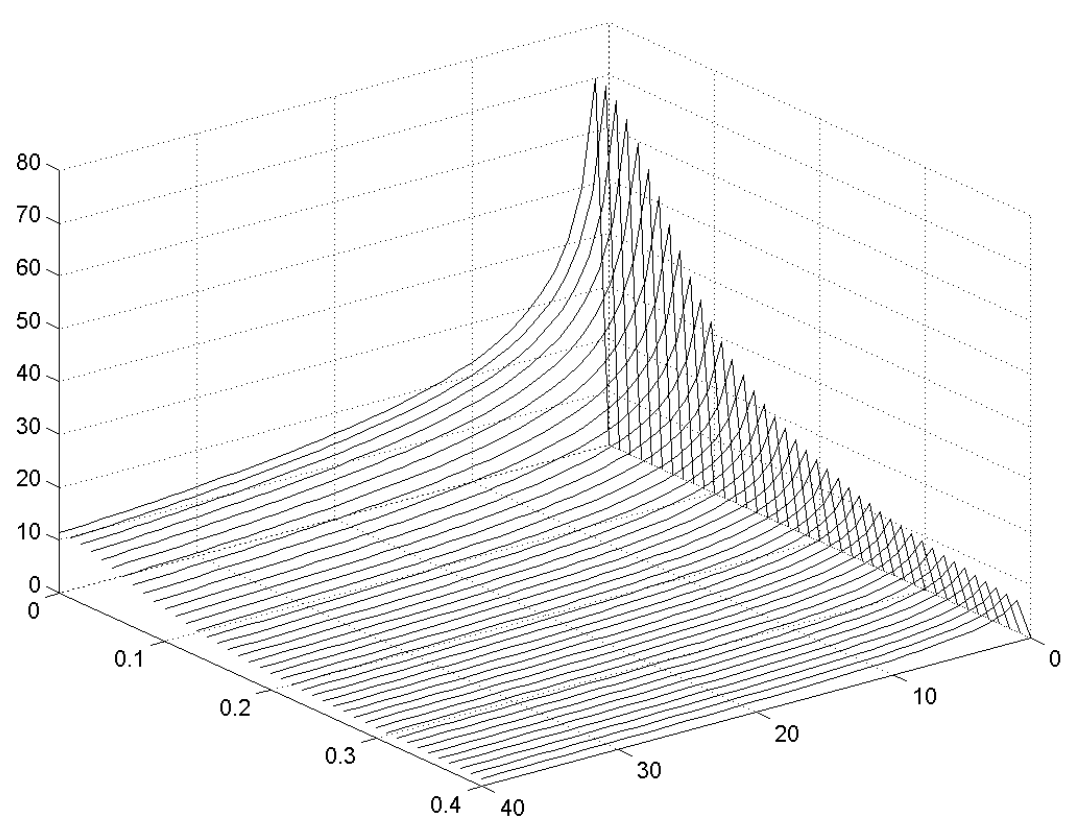

Figure 9: The temperature distribution along the drill depth in dependence of the time

thermal couple inside the top of the drill bit during a real experiment, reported in [3]. Really, the horizontal axes there are the drill depth and the drill speed except the time but the graphics is topologically similar to the one on the fig.9. As a conclusion it could be said that the correspondence of temperature values with the values related to the drill depth and the time could be established by calculations based on a measurement not on the top of the drill bit (inside the bone) but on the bone surface (where the drill bit goes into the bone while it is moving deeply inside). The real experimental measurements of the temperature inside the bone, published in [3], could be considered as a verification of the theoretical state. 


\section{Discussions and Conclusions}

Automatic bone drilling can solve problems arising during the usual manual drilling. The proposed experimental setup was designed to identify certain characteristics of the bone drilling process such as resistant force owing to variable bone density, appropriate mechanical torque during the manipulation, linear speed and the electromechanical parameters of motors and drives. Automatic drilling must also guarantee a patient's safety, i.e., it should avoid bone damage due to overheating and substantial translation stress.

The usage of ultrasonic vibration device for ultrasonically-assisted drilling of a cortical bone, which is reported in [10], shows that the resistant force is reduced in some extent. But the experiment is executed handily and the vibrations have constant frequencies and amplitudes, i.e. they cannot be controlled. The controlled trust force application is the higher step on the way of the resistant force reduction which finally reflects on the temperature reduction. At last, that can be realized by automatic drilling execution only, which proves another advantage of robot application in the orthopaedic surgery

The experiments also disclosed a solution to the overheating problem. A plug-in temperature sensor was mounted in the experimental setup to guarantee temperature recording before, during and after drilling. Our results showed that a small variation in the drilling parameters yields a temperature increase. The total drilling time never exceeds $20 \mathrm{~s}$, really $6-10 \mathrm{~s}$ and the maximal temperature is less than $50^{\circ} \mathrm{C}$. That is in agreement with the result mentioned above that the bone must not be exposed for longer than $30 \mathrm{~s}$ at $50^{\circ} \mathrm{C}$. The comparison between hand and automatic drilling shows the controlled trust force almost twice decreases the resistant force reflecting to the temperature reduction, which can be realized in automatic drilling mode only. The precise drilling performance proves the advantage of using drilling robots in surgery, assuring in the same time more reliability and safety.

\section{Acknowledgments}

The support of the Bulgarian Ministry of Education and Science, Grant FFNNIPO_12_01547 is acknowledged. 


\section{References}

[1] J. Lundskog, Heat and bone tissue, Scand. J. Plastic and Reconstructive Surgery, Sup. 9 (1972).

[2] R. Huskies, Some fundamental aspects of human joint replacement, Acta Orthop. Scand., 185 (1979), 62-63.

[3] M.T. Hillery, I. Shuaib, Temperature effects in the drilling of human and bovine bone, Journal of Materials Processing Technology, 92-93 (1999), 302-308.

[4] A.R. Mortiz, F.C. Henrique, Studies of thermal injuries, II, Am. J. Pathol., 23 (1947), 695.

[5] A.R. Mortiz, F.C. Henerique, The relative importance of time and surface temperature in the causation of cutaneous burns, Am. J. Physiol., 23 (1947), 695-719.

[6] K.L. Wiggins, S. Malkin, Drilling of bone, J. Biomech, 9 (1976), 553-559.

[7] L.S. Methews, C.A. Green, The thermal effects of skeletal fixation Pin insertion in bone, J.B.J.S., 66A, No. 1 (1984).

[8] C. Natali, P. Ingle, J. Dowell, Orthopedic bone drills can they be improved? Temperature changing near the drilling face, J. of British Editorial Society of Bone and Joint Surgery, 78B, No. 3 (1996), 357-362.

[9] G. Augustin, et al., Cortical bone drilling and thermal osteonecrosis, Clin. Biomech. (2011), doi: 10.1016/j.clinbiomech.2011.10.010.

[10] K. Alam, A.V. Mitrofanov, V.V. Silberschmidt, Experimental investigations of forces and torque in conventional and ultrasonically-assisted drilling of cortical bone, Medical Engineering 83 Physics, 33 (2011), 234239.

[11] S. Sarakankosol, W. Charoensuk, J. Suthakorn, Development of the microVibrated Robot for orthopedic surgery in drilling application, In: The 7-th Asian Conference on Computer Aided Surgery (ACCAS 2011).

[12] G. Boiadjiev, T. Boiadjiev, V. Vitkov, K. Delchev, R. Kastelov, K. Zagurski, Robotized system for automation of the drilling in the orthopedic surgery. Control algorithms and experimental results, In: Proceedings of the 9-th IFAC Symp. on Robot Control SYROCO'09, Gifu, Japan (2009), 633-638. 
592 G. Boiadjiev, K. Delchev, T. Boiadjiev, K. Zagurski, R. Kastelov, V. Vitkov

[13] T. Boiadjiev, K. Zagurski, G. Boiadjiev, K. Delchev, V. Vitkov, I. Veneva, R. Kastelov, Identification of the bone structure during the automatic drilling in the orthopedic surgery, J. of Mechanics Based Design of Structures and Machines, 39, No. 2 (2011), 285-302.

[14] G. Boiadjiev, R. Kastelov, T. Boiadjiev, V. Kotev, K. Delchev, K. Zagurski, V. Vitkov, Design and performance study of an orthopaedic surgery robotized module for automatic bone drilling, Journal of Medical Robots and Computer Assisted Surgery (2013), doi: 10.1002/rcs.1479.

[15] JuFun Lee, A new thermal model for bone drilling with applications to orthopaedicsurgery, Medical Engineering and Physics, 33 (2011), 12341244, doi: 10.1016/j.medengphy.2011.05.014.

[16] V. Smirnov, Equations with Partial Derivatives, Vol. 4, Moskow (1982).

[17] G. Boiadjiev, R. Kastelov, T. Boiadjiev, D. Vassileva, Robot application in medicine for orthopaedic drilling manipulation, In: The 8-th Mechatronics Forum International Conference on Mechatronics, 2002, Twente, Netherlands. (CD), ISBN 9036517664, Book of Abstracts, 87, ISBN 9036517672.

[18] Patent No 1053 / 24.07.2008, BG.

[19] Patent No 66136/17.08.2011, BG. 